\title{
Tissue-Specific Expression from a Compound TATA-Dependent and TATA-Independent Promoter
}

\author{
PAUL A. GARRITY AND BARBARA J. WOLD* \\ Division of Biology, 156-29, California Institute of Technology, Pasadena, California 91125
}

Received 7 June 1990/Accepted 7 August 1990

\begin{abstract}
We have found that the mouse metallothionein-I (MT-I) gene promoter functions in an unusual, compound manner. It directs both TATA-dependent and TATA-independent modes of transcription in vivo. The TATA-dependent message is initiated at the previously characterized +1 transcription start site and is the predominant species in most tissues. In many cell types it is metal inducible. The TATA-independent initiation sites are distributed over the 160 bp upstream of the previously characterized +1 start site, and the RNA products are present in all tissues examined. Only in testis, however, do the TATA-independent transcripts predominate, accumulating to highest levels in pachytene-stage meiotic cells and early spermatids. Unlike the TATA-dependent +1 transcript, these RNAs are not induced by metal, even in cultured cells in which the +1 species is induced. Transfection studies of site-directed mutants show that destruction of the TATA element drastically alters the ratio of the two RNA classes in cells in which the +1 transcripts normally dominates. In TATA-minus mutants, the TATA-independent RNAs become the most prevalent, although they remain refractory to metal induction. Thus, the MT-I promoter utilizes two different types of core promoter function within a single cell population. The two different types of core promoters respond very differently to environmental stimuli, and the choice between them appears to be regulated in a tissue-specific fashion.
\end{abstract}

Two different classes of promoter structure and function have been described for RNA polymerase II transcription in eucaryotes. The first class includes the majority of genes and is characterized by the presence of a TATA element which specifies the site of transcription initiation, usually about 30 nucleotides downstream. Extensive genetic and biochemical evidence has shown that the TATA element plays a significant role in start site selection and in specifying the frequency of transcriptional initiation $(3,5,12,15,17,19,22$, $25,40)$. By itself, a TATA element is sufficient to stimulate specific transcription in vitro, though it does so with low efficiency in vivo in the absence of auxilliary elements (32, 45). Based on these properties, the TATA element can reasonably be considered to constitute a type of core promoter. The activity of such a core promoter is dependent in vivo on interactions with other protein-DNA complexes. These modulators include transcriptional enhancers, which can act at considerable distances from the core promoter, as well as more proximal upstream elements that are less flexible than enhancers in their orientation and distance requirements (38). In the current view, enhancers and upstream elements mediate environmental and developmental regulation of transcription initiation by positively or negatively modulating the activity of the core promoter complex $(25,38)$.

A second class of core promoters lacks a detectable TATA element. Some of these initiate transcription at a single position (50), while others initiate transcription at multiple sites over an extended region $(16,26,31,35,37,44,47)$. The nature and extent of mechanistic differences between TATA-dependent and TATA-independent modes of transcription initiation are not presently known. In genes described to date, a promoter has been considered either TATA dependent or TATA independent, with developmen-

\footnotetext{
* Corresponding author.
}

tal regulation determined by the action of separable positive or negative modulators on this core promoter structure (or in some cases more than one core promoter of the same type, e.g., two TATA elements [51]). Here we describe an interesting departure from this view of fundamental promoter classes and their developmental regulation based on the study of promoter structure and function for the mouse metallothionein-I (MT-I) gene.

The MT-I gene is constitutively expressed in many tissues and can be induced to higher levels by exposure to heavy metals (13). This induction has a major transcriptional component (13) and can be attributed in large part to the positive action of upstream metal-regulatory elements (MREs) on the TATA-dependent core promoter $(7,49,52)$. Here, closer examination shows that there is also a non-metal-inducible population of MT-I RNAs that are initiated upstream of the TATA element and, at least in cultured fibroblasts, are TATA independent. Thus, the MT-I promoter simultaneously uses both TATA-dependent and TATA-independent transcription regimes which are divergent in their response to environmental stimuli. Moreover, there appears to be profound tissue-specific regulation in the choice of core promoter mechanism. Upstream RNAs similar to those seen in fibroblasts and many other cell types are the predominant species in the testis. Testis has previously been noted as unique among the tissues which have been studied in its regulation of MT-I RNA. Despite possessing a high basal level of MT-I RNA, it does not respond to cadmium (Cd) accumulation with a rapid increase in MT-I transcripts (13). Thus, MT-I is a naturally occurring case of compound core promoter function, and the choice between the modes of transcriptional initiation appears to be regulated in a tissuespecific fashion. Previously, this had not been considered a choice but rather an intrinsic property of gene architecture. These observations contribute to a revised view of the interaction of regulatory elements, including MREs, with the two classes of initiation complex. 


\section{MATERIALS AND METHODS}

Constructs and probes. Standard procedures and manufacturer's instructions were used in constructing plasmids and synthesizing RNA transcripts. Four MT-I probes were used. The 5'-end genomic probe was generated from pSP6-MT-I (gift of P. Mueller), which contains mouse MT-I genomic sequences in the pSP64 vector. pSP6-MT-I was cut with $B s t$ EII and transcribed with SP6 RNA polymerase. pMT-I $\Delta \mathrm{i}$ (gift of R. D. Palmiter) was used to create pMT-ISH and pMT-IBH. pMT-ISH was created by inserting a SacI-HindIII fragment of pMT-I $\mathrm{i}$ into SacI- and HindIIIcut pT3/T7-19 (Bethesda Research Laboratories, Inc.). pMT-ISH was cut with EcoRI and transcribed with T7 RNA polymerase to generate the full-length cDNA probe. pMTIBH was created by inserting a BglII-HindIII fragment of pMT-I $\Delta \mathrm{i}$ into BamHI- and HindIII-cut pBluescriptSK ${ }^{+}$ (Stratagene). pMT-IBH was cut with $X b a I$ and transcribed with T3 RNA polymerase to generate the $3^{\prime} \mathrm{cDNA}$ probe. pMT-INT (gift of P. Mueller), a BglII-HinPI fragment of pMT-I $\Delta \mathrm{i}$ inserted in BamHI- and $A c c \mathrm{I}-$ cut pT3/T7-19, was cut with $E c o$ RI and transcribed with T7 RNA polymerase to generate the internal cDNA probe. $\mathrm{pBS}^{-}$(Stratagene) was a gift of S. Tavtigian. pMTCAT (30) is a derivative of pBluescriptKS ${ }^{-}$(Stratagene), which contains the MT-I promoter EcoRI-BglII fragment, the chloramphenicol acetyltransferase (CAT) sequence of pSV2cat, and the simian virus 40 splicing and poly(A) addition signals. Oligonucleotide-directed mutagenesis was used to create pMCSPA (gift of L. J. Maher and S. J. Salser), the Sp1 site mutant derivative of pMTCAT, and pMCT, the TATA mutant derivative of pMTCAT. The substitution of TCGAGA for the wild-type TATAAA sequence in pMCT was anticipated to disable TATA element function. The double mutant TAGAGA has previously been shown, in studies in which the TATA-binding transcription factor TFIID from Saccharomyces cerevisiae was used to complement a mammalian transcriptional extract, to drastically reduce transcription from the adenovirus type 2 major late promoter in vitro (6). Furthermore, the binding affinity of yeast TFIID for the double mutant adenovirus major late promoter is similar to its affinity for nonspecific DNA (21).

Cell culture and induction. $P$ cells (derived from $L$ cells as described in reference 29) were maintained in dialyzed calf serum (39). Eight hours prior to harvesting for RNA, fresh medium with or without $6 \mu \mathrm{M} \mathrm{CdSO}$ was added. Cells for transfection were plated at $3.7 \times 10^{5}$ per $10-\mathrm{cm}$ dish and transfected by a standard calcium phosphate coprecipitation $27 \mathrm{~h}$ later. Each plate received $10 \mu \mathrm{g}$ of test plasmid (pMTCAT, pMCT, pMCSPA, or $\mathrm{pBS}^{-}$), $10 \mu \mathrm{g}$ of $\mathrm{L}$ cell DNA, and $1 \mu \mathrm{g}$ of $\mathrm{pY} 3$, which confers resistance to hygromycin B. After $11 \mathrm{~h}$ of DNA exposure, cells were refed with fresh medium. After $24 \mathrm{~h}$, cells were refed with fresh medium containing $200 \mu \mathrm{g}$ of hygromycin B per ml. The cells were refed every 3 days with fresh selection medium until they had spent 17 days in selection medium. More than 90 separate colonies were obtained on each plate. Each test plasmid was used to transfect three plates, and the pools obtained were maintained and analyzed separately. No viable colonies were recovered from a control plate which had received no DNA. Cells were split three times prior to RNA analysis and were maintained in selection medium. Eight hours prior to harvesting for RNA, fresh hygromycin-

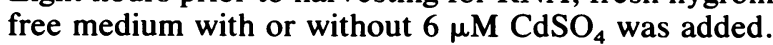

Animals and induction. Male mice (8 to 15 weeks old) of strains DBA/2J, C57BL/6J (both obtained from Jackson
Laboratories, Bar Harbor, Maine) and $\mathrm{BDF}_{1}$ (offspring of DBA $\times$ C57BL matings) were used. No strain or age variation in MT RNA expression was noted, and 15-weekold DBA/2J mice were used for quantitation. Mice were injected subcutaneously with $200 \mu \mathrm{l}$ of $\mathrm{H}_{2} \mathrm{O}$ (or $0.9 \% \mathrm{NaCl}$ ) with or without $\mathrm{CdSO}_{4}(10 \mathrm{mg}$ of $\mathrm{Cd}$ per $\mathrm{kg}$ of mouse body weight) $3 \mathrm{~h}$ before the mice were sacrificed by cervical dislocation. Tissues were flash-frozen in liquid nitrogen and stored at $-80^{\circ} \mathrm{C}$ until RNA was isolated.

Pretreatment of mice with $\mathrm{Cd}$ was as described in reference 42, using $\mathrm{CdSO}_{4}$ instead of $\mathrm{CdCl}_{2}$. Bodies, testes, and kidneys were weighed after the mice were sacrificed. No body or organ weight differences were noted between mice pretreated with daily injections of saline $(n=5)$ and those receiving saline with $\mathrm{CdSO}_{4}(0.25 \mathrm{mg}$ of $\mathrm{Cd}$ per $\mathrm{kg})(n=6)$ after 12 days. The testes of mice given a single injection of 1 $\mathrm{mg}$ of $\mathrm{Cd}$ per $\mathrm{kg}$ after pretreatment with saline $(n=6)$ and examined 13 days later weighed approximately half as much as those from mice injected with saline after the saline pretreatment $(n=5)$. There was no effect on body or kidney weight. Mice pretreated with $\mathrm{CdSO}_{4}$ showed no such decrease in testicle weight $(n=4)$.

RNA preparation. RNA was prepared from $L$ cell derivatives ( $P$ cells) as described before $(11,39)$. RNA was prepared from mice as described in reference 11 , with the addition of a second organic extraction. Purified testis cell type and mutant mouse RNAs were provided by Kelly Thomas and Mel Simon (sample set 1 [reference 53]) and by Debra Wolgemuth (sample set 2 [reference 54]). These RNAs were prepared by $\mathrm{LiCl}$ precipitation (9). RNA concentrations were determined by the $A_{260}$. DNase treatment of RNAs did not alter the results.

RNase protection. RNase protection assays were performed as described before $(29,56)$ with the following modifications. RNA probes were isolated prior to use from either $N, N^{\prime}$-bis-acrylylcystamine acrylamide (Bio-Rad Laboratories) or conventional acrylamide gels. The type III preparation of small yeast RNAs (Sigma catalog no. R-7125) was used as carrier RNA and in lanes labeled tRNA control. Total MT-I RNA levels were determined by using the 5' genomic probe and the internal cDNA probe. Protected bands were excised from the gel, and the radioactivity was counted. Counting efficiency was determined by counting gel-isolated dilutions of probe. Transcript abundances were also determined from autoradiographs of $5^{\prime}$ genomic probe RNase protection experiments and MT-I oligonucleotide primer extensions (see below) with an LKB XL densitometer. The densitometric results were generally consistent with the results from scintillation counting.

Primer extensions. Primer extensions were performed with avian myeloblastosis virus reverse transcriptase (Life Sciences) (27). Reverse transcription of endogenous MT-I RNA was primed with an oligonucleotide (5'-CGGAGTAAGT GAGGAGAAGGTACTC-3') complementary to nucleotides +45 through +23 . A 0.1-pmol amount of gel-isolated, 5 '-endlabeled primer was used in each reaction mixture. Signal linearity was confirmed by analyzing dilutions of a single RNA sample. The results of RNase protection experiments carried out on all samples in Fig. 1C (Fig. 1B and data not shown) indicate that the primer extension products in Fig. $1 C$ accurately reflect MT-I RNA termini. Primer extension products were quantitated by densitometry as above. Reverse transcription of MT-CAT fusion RNAs was primed with an oligonucleotide (5'-ATATCAACGGTGGTATATCC AGTGA-3') complementary to CAT sequences. It lies 143 to 


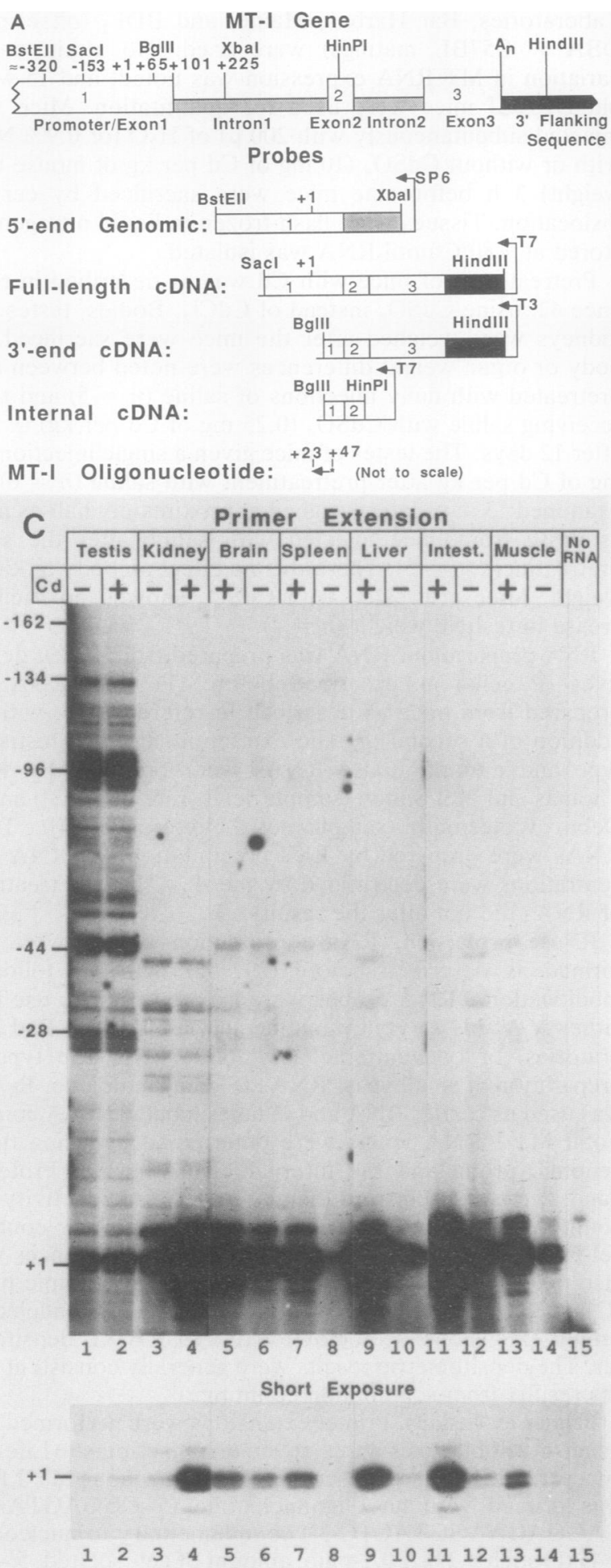

119 bp downstream of the MT-I +1 site in the pMTCAT plasmid.

\section{RESULTS}

Expression pattern of the multiple species of MT-I RNA. MT-I RNA was examined in tissues from DBA/2J, C57BL/ $6 \mathrm{~J}$, and $\mathrm{BDF}_{1}$ mice, in NIH $3 \mathrm{~T} 3$ cells, and in an $\mathrm{L}$ cell

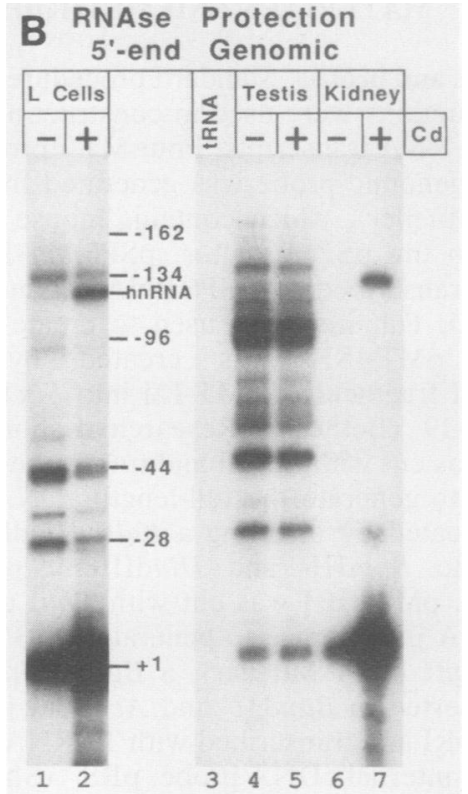

FIG. 1. (A) Structure of the mouse MT-I gene and the antisense probes used in RNase protection and primer extension assays. Sequence is from reference 18, with an additional A at -112 (39). Unshaded boxes, Exon and promoter sequences; stippled boxes, introns; black boxes, sequences downstream of $3^{\prime}$ cleavage and polyadenylation site $\left(A_{n}\right)$; thin horizontal lines, vector sequences. Restriction sites are numbered according to complementary nucleotides retained in derived probes. (B) RNase protection analysis performed with 5 '-end genomic probe. Sample RNA $(8 \mu \mathrm{g})$ plus tRNA $(30 \mu \mathrm{g})$ were used in lanes 1,2 , and 4 to $7 ; 30 \mu \mathrm{g}$ of tRNA alone was used in lane 3 . Products were initially sized by using DNA markers and subsequently assigned to specific start sites by comparison with primer extension results. Longer exposures show upstream RNAs present in the kidney. The smear near -28 in lane 7 is artifactual. Please note that because RNase protection probes are continuously labeled, the specific activity (disintegrations per minute per mole) of each protected product increases with product size. hnRNA, heterogeneous nuclear RNA (unspliced + 1 RNA). (C) Primer extensions performed with the MT-I oligonucleotide. An 8- $\mu \mathrm{g}$ amount of RNA was used in each lane (except lane 15, to which no RNA was added). Product identities were determined by comparison with adjacent lanes generated by dideoxy sequencing of MT-I with the same oligonucleotide. Subsequent analysis of multiple independent preparations of RNA indicated that the apparent metal induction of upstream RNA in liver shown in this figure was anomalous and that upstream RNAs in liver were actually slightly repressed by metal. Intest., Intestine.

derivative ( $\mathrm{P}$ cells [29]) by RNase protection (Fig. 1B and data not shown) and primer extension (Fig. 1C). The profile of MT-I RNA in testis (Fig. 1B, lanes 3 and 4, and Fig. 1C, lanes 1 and 2) was dramatically different from that found elsewhere. The expected transcript, with its $5^{\prime}$ end at the previously described +1 site (18), constituted only $15 \%$ of the total MT-I RNA in testis. The remainder were transcripts that extended well upstream. (To facilitate quantitation, we have operationally grouped the many species of transcripts into six major groups, as indicated in Fig. 2B. Total MT-I and individual start site RNA data are presented in the Fig. 2A histograms.) Although this $5^{\prime}$ heterogeneity was most striking in the testis, close inspection of both primer extension and RNase protection experiments showed that all sources tested possessed a complex array of MT-I 5' ends. For example, L cells, while producing high levels of the +1 
A

\section{Total MT-I RNA}

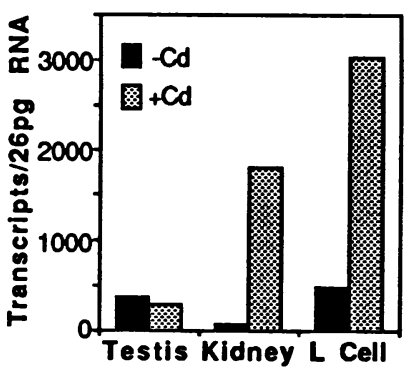

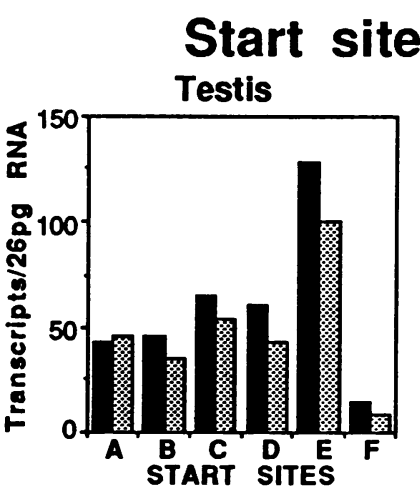

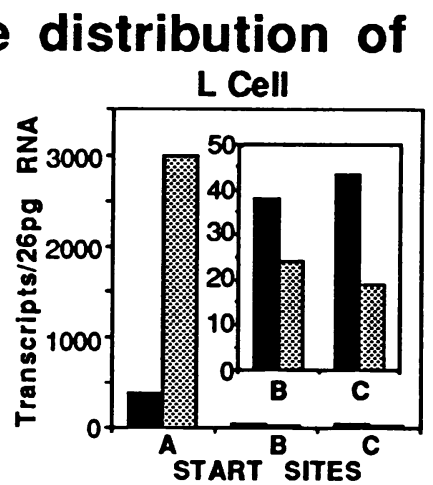

MT-I RNA

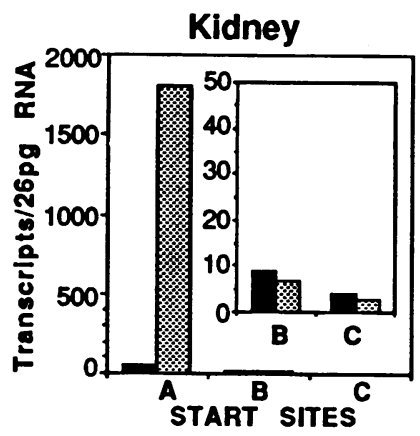

B

\section{MT-I promoter and transcript start sites}

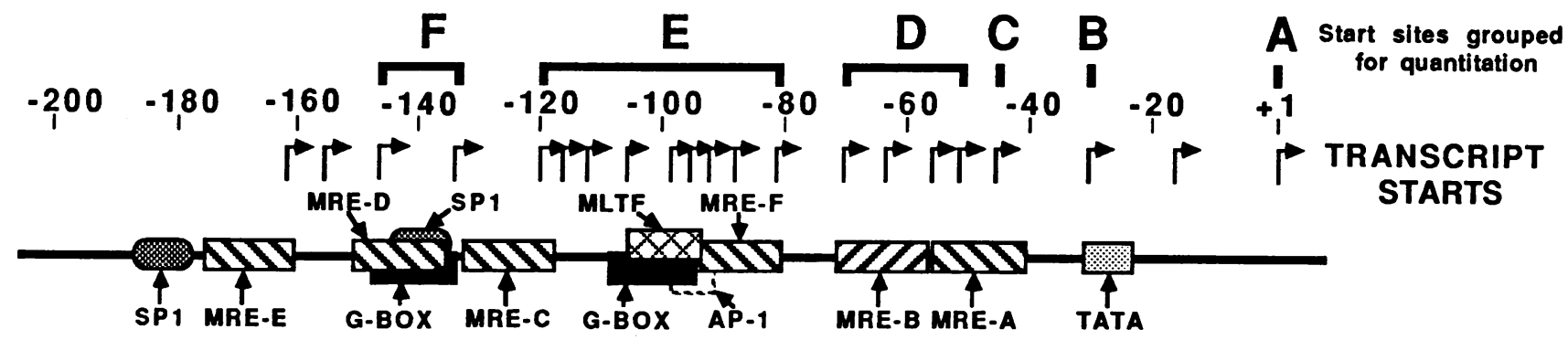

FIG. 2. (A) Transcripts per $26 \mathrm{pg}$ of RNA, the amount of RNA in an L cell (4). The quantitation procedure is described in Materials and Methods. Transcriptional start sites were grouped into six clusters to ease analysis (see panel B). RNA termination sites by group: A, + 3 to $-1 ; \mathrm{B},-25$ to $-35 ; \mathrm{C},-39$ to $-45 ; \mathrm{D},-51$ to $-75 ; \mathrm{E},-82$ to $-119 ; \mathrm{F},-133$ to -146 . (B) Map of MT-I promoter, with arrows indicating the position of each discrete start site (judged by its appearance as a unique entity in both RNase protection and primer extension experiments) and with the groupings used in quantitation indicated. Groupings reflect the smallest regions which could be reliably excised from a gel and/or densitometrically integrated. Boxes indicate potential DNA-protein interaction sites (39). The MREs show metal-inducible dimethyl sulfate (DMS) protection in L cells in vivo. The distal Sp1 site binds Sp1 in vitro and shows an Sp1-like footprint in L cells in vivo. The proximal Sp1 site binds Sp1 weakly in vitro but does not shown an Spl-like footprint in L cells in vivo. The MLTF site shows MLTF-like DMS protection in L cells in vivo. The G element (G-BOX) is a conserved sequence feature of the MT gene family with no known function. The potential AP-1 site (1) has not been shown to interact with this factor.

transcript, expressed essentially the same set of upstream RNAs as the testis, although many of these species (e.g., -96) were expressed at much reduced levels (Fig. 1B, lanes 1 through 5, and Fig. 2), while kidney expressed lower levels of upstream RNA than either testis or L cells and used a different set of upstream initiation sites (Fig. 1B, lanes 6 and 7; Fig. 1C, lanes 3 and 4; and Fig. 2). Inspection of the MT-I promoter sequence revealed no other TATA homologies in this region and no concordance between the upstream start sites and potential "initiator" elements of the type shown to fix the start site in the TATA-less mouse terminal deoxynucleotidyltransferase gene (50). The MT-I upstream DNA also lacked any identifiable HIP1-binding sequences, which direct start site selection at one of the several start sites in the mouse dihydrofolate reductase (dhfr) gene (33).

Cd administration induced only the +1 RNA species. The +1 RNA levels increased by at least $40 \%$ and commonly over 25 -fold in all cells and tissues surveyed except testis (Fig. 1C, short exposure), in agreement with previous determinations of total MT-I RNA content (13). Steady-state levels of the upstream MT-I RNAs were relatively unaffected by $\mathrm{Cd}$. The greatest change in the upstream RNAs was detected in $\mathrm{L}$ cells, where the longer transcripts decreased 40 to $60 \%$ after metal treatment (Fig. 1B, lanes 1 and 2; Fig. 2); testis and kidney showed smaller but reproducible diminutions (Fig. 1B, lanes 4 and 5; Fig. 1C, lanes 1 through 4; Fig. 2). These upstream, non-metal-inducible transcripts were a specific property of MT-I rather than a ubiquitous feature of metallothionein genes. In contrast to MT-I, the closely related mouse metallothionein-II (MT-II) (48) transcripts showed little upstream heterogeneity at the $5^{\prime}$ end and exhibited metal induction behavior paralleling that of the MT-I + 1 RNA (data not shown).

The many species of MT-I RNA differ only at their site of initiation. We examined MT-I RNA from various sources to see whether the upstream initiation sites produced RNAs substantially different from the traditional +1 site RNAs. RNA gel blots, containing samples from testis and kidney, were probed with an oligonucleotide specific for upstream RNA and with an oligonucleotide which would hybridize to both upstream and +1 RNAs. In each experiment only one general size species of MT-I RNA was detected (data not shown), indicating no gross structural differences in the body of the RNA. More sensitive assays for structural heteroge- 


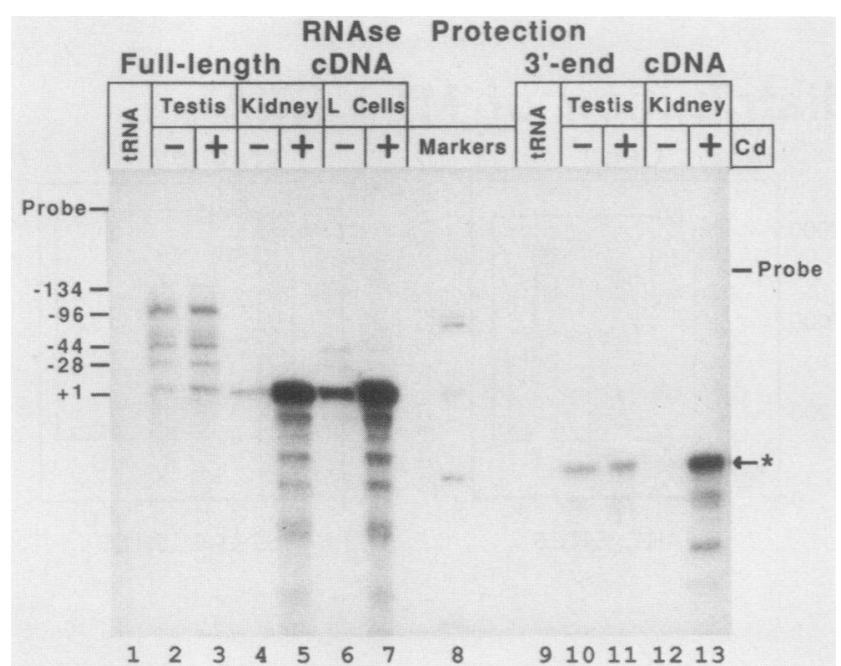

FIG. 3. RNase protection assays performed with the full-length cDNA probe (lanes 1 to 7) and 3'-end cDNA probe (lanes 9 to 13 ). Sample RNA $(3.4 \mu \mathrm{g})$ plus tRNA $(30 \mu \mathrm{g})$ was used in lanes 2 to 7 and lanes 10 to $13 ; 30 \mu \mathrm{g}$ of tRNA alone was used in lanes 1 and 9 . Products were sized by using HpaII-cut pBR322 and pUC19 (lane 8 ). Products were assigned to specific start sites based on comparison with Fig. 1. Longer exposures showed upstream RNAs present in the kidney. Note that the complementarity of the full-length cDNA probe extended only to -153 . The asterisk denotes the predicted protection product of the 3 '-end cDNA probe.

neity were also performed. 3 '-End cDNA and full-length cDNA probes (Fig. 1A) were used in RNase protection assays. The 3 '-end cDNA probe (Fig. 3, lanes 9 to 13 ) required appropriate splicing and $3^{\prime}$ cleavage of the RNA to yield the product indicated by the asterisk in Fig. 3. The amounts of this product in testis and kidney were consistent with our total MT-I RNA measurements and so represented the vast majority of MT-I RNA species. Furthermore, the full-length cDNA probe (Fig. 3, lanes 1 to 7 ), which is sensitive to the transcription initiation site as well as splicing and 3' cleavage, recapitulated the start site distribution seen in RNase protection with the $5^{\prime}$-end genomic probe (Fig. 1B, lanes 1 to 7) and in primer extension with the MT-I oligonucleotide (Fig. 1C, lanes 1 to 4). These data, combined with previous work establishing that all MT-I RNA in testis is polyadenylated (55), show that, within our limits of detection, all species of MT-I transcripts are spliced and 3 '-end processed identically and therefore differ from one another only at their $5^{\prime}$ ends.

On the basis of the data presented thus far, it was formally possible that the upstream RNAs could be hitherto-undetected precursors of the +1 species that accumulate to higher steady-state levels in testis. Such a pattern of 5 '-end processing would be unprecedented, however, and appears unlikely to be the case for two reasons. First, the longer RNAs persisted in $\mathrm{L}$ cells over the course of a 6-h dactinomycin (actinomycin D) transcriptional block instead of being chased into the +1 form (or any other detectable form) by subsequent processing (data not shown). Also, as detailed below, site-directed mutation of the MT-I TATA element virtually eliminated the +1 product while leaving upstream RNAs intact, an observation inconsistent with a precursorproduct relationship.

Testis sensitivity to $\mathrm{Cd}$ can be altered without changing MT RNA behavior. Metallothionein proteins bind heavy metals (like $\mathrm{Cd}$ ) and can protect cells from their potentially toxic effects (23). Compared with other tissues, testis shows an unusual sensitivity to $\mathrm{Cd}$, suffering widespread and irreversible damage at $\mathrm{Cd}$ doses that have no other major effect on the animal $(34,43)$. However, treatment of susceptible strains with low doses of $\mathrm{Cd}$ protects these mice from testicular damage by a subsequent high dose of $\mathrm{Cd}$ (42). We reexamined these phenomena in the light of our present knowledge of MT RNA behavior in testis. It is possible that testis hypersensitivity might be due to the noninducibility of MT-I and MT-II RNAs in testis and that this noninducibility might be related to the unusual behavior of MT-I RNA there. A mouse strain reported to be "less susceptible" to testicular damage by $\mathrm{Cd}(20), \mathrm{C} 57 \mathrm{BL} / 6 \mathrm{~J}$, was examined and showed no difference in basal or induced expression of MT-I and MT-II (data not shown) compared with the susceptible strain $\mathrm{DBA} / 2 \mathrm{~J}$. A low-level $\mathrm{Cd}$ pretreatment regime did protect DBA/2J mice from $\mathrm{Cd}$-induced testis damage but had no discernible effect on the basal level of MT-I RNA or MT-II RNA in testis, nor did it render these genes metal inducible in this tissue (data not shown). Thus, it is possible to change Cd sensitivity without modifying MT RNA expression. Although the failure of MT RNA to be induced by metal in testis may contribute to the organ's susceptibility to $\mathrm{Cd}$ poisoning, additional mechanisms are involved in generating resistance by the $\mathrm{Cd}$ pretreatment regime.

Upstream MT-I RNAs are the predominant species in germ cells of the testis. The testis is a complex organ containing specialized somatic accessory cells, such as Sertoli and Leydig cells, along with premeiotic germ cells, cells in various stages of meiosis, and maturing sperm. To find out which of these very different cell types are responsible for the predominance of upstream MT-I transcripts in total testis, RNAs from fractionated cell populations from the testes of mice not exposed to metal were first examined. The upstream RNAs were most prominent in late meiotic prophase (pachytene-stage cells) and remained prominent in the early stages of sperm maturation (round spermatids) (Fig. 4, lanes 3 to 7). Thus, in germ cells, the level of upstream RNA per unit mass of total RNA increased late in meiosis and then declined in the residual bodies and cytoplasmic fragments cast off by condensing spermatids (Fig. 4, lanes 1 to 7). Conversely, the level of the +1 RNA gradually declined as the germ cell population proceeded from spermatogonia to round spermatids and was even lower in the residual bodies and cytoplasmic fragments.

Another method, independent of cell separation techniques, can distinguish expression in germ cells from expression in accessory cells. Mutant mice of the $\mathrm{W} / \mathrm{W}^{v}$ genotype lack germ cells but possess all the somatic cell types of the testis $(36,41,55)$. The testes of $\mathrm{W} / \mathrm{W}^{v}$ mice were found to contain substantially lower levels of upstream RNA than the phenotypically wild-type testes of $+/ \underline{W}^{v}$ mice (Fig. 4 , lanes 9 and 10). This argues that high-level upstream MT-I transcript expression is limited to germ cells.

Can the different species of MT-I RNA encode variant proteins? Inspection of the 5' nucleotides of the longer transcripts indicated that they contained an open reading frame that could code for a 22 -amino-acid protein (showing none of the cysteine-richness of the MTs) after initiation at the -91 ATG. However, there were stop codons in this and all other reading frames between -4 and +40 , upstream of the previously recognized initiation codon. It therefore appears unlikely that the extra nucleotides contribute any information toward a larger than usual MT protein. Possible effects of the longer 5' leader on RNA processing efficiency, 


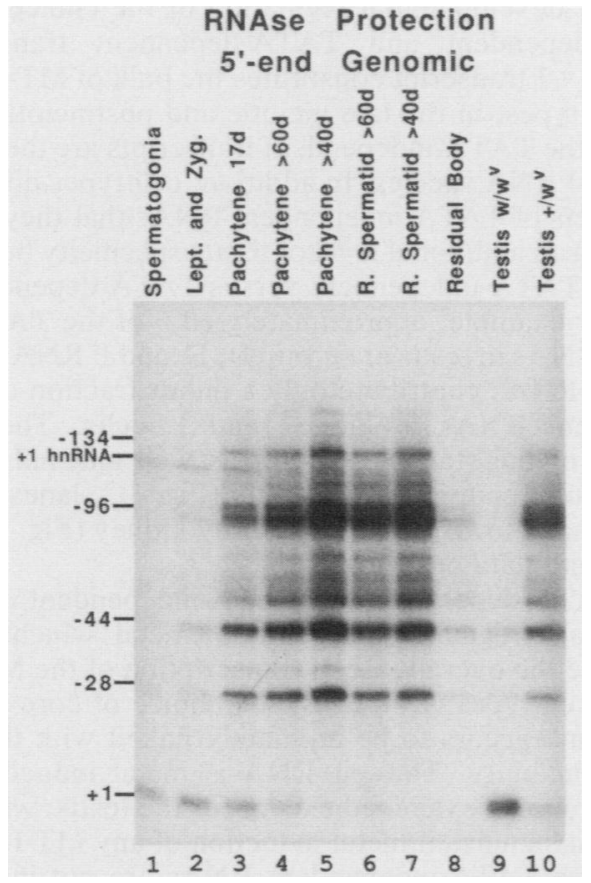

FIG. 4. RNase protections performed with the 5 '-end genomic probe. Sample RNA $(0.5 \mu \mathrm{g})$ plus tRNA $(30 \mu \mathrm{g})$ was used in each lane. The tRNA lane (not shown) gave no signal. Mouse age at preparation of sample: type A and B spermatogonia, 8 days; leptotene (Lept.) and zygotene (Zyg.) cells, 17 days; pachytene and round (R.) spermatids as indicated (in days); residual body and mutant mouse testes, $>40$ days. Lanes $1,2,3,4$, and 6 are from sample set 1 . Lanes $5,7,8,9$, and 10 are from sample set 2 . The practical necessity of using mice of different ages to prepare the various cell populations did not appear to compromise our conclusions about stage specificity. The MT-I RNA profiles of pachytene cells from 17-, $>60$-, and $>40$-day-old mice were qualitatively similar, while the leptotene, zygotene, and pachytene cells from 17-day-old mice were quite different. hnRNA, heterogeneous nuclear RNA (unspliced +1 RNA).

message stability, or translational regulation remain to be examined.

Site-directed mutation separates MT-I RNA species into TATA-dependent and TATA-independent classes. The presence of a group of RNAs that initiate upstream of the TATA homology element and are regulated differently than the +1 RNA suggested that the MT-I promoter might be directing transcription in two different ways, one TATA dependent and the other TATA independent. We directly tested this possibility by constructing appropriate mutants of the MT-I promoter and analyzing the RNA products of each in transfection assays. Because $L$ cells express the same overall repertoire of upstream start sites as germ cells (albeit with quantitative variations) (Fig. 1B), they were used as transfection hosts. The constructs contained MT-I sequence extending from $1.7 \mathrm{~kb}$ upstream to $65 \mathrm{bp}$ downstream of the MT-I +1 site fused to the CAT gene (30). Site-directed mutagenesis was used to disrupt the TATA element by substitution of the sequence TCGAGA for the wild-type TATAAA. A construct containing mutations in the distal Sp1 site (GGGGCGG changed to GCCCGGG) was also created. This $\mathrm{Sp} 1$ site binds $\mathrm{Spl}$ in vitro, is occupied in $\mathrm{L}$ cells in vivo (39), and is of interest because $\mathrm{Sp} 1$ activates TATA-independent transcription from the $d h f r$ promoter in vitro (14).

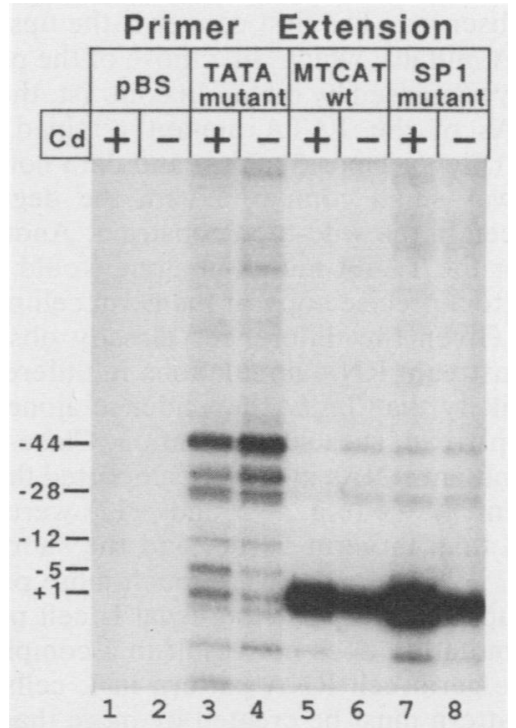

FIG. 5. Primer extensions performed with the transgene-specific MT-CAT oligonucleotide. A 9- $\mu$ g amount of RNA from each transfected cell line was used per lane. Overexposure of the autoradiograph necessary to see upstream RNA and mutant +1 RNA caused the induction of the +1 RNA in the wild-type (wt) and Spl mutant lanes to be underrepresented. Product identities were assigned by comparison with adjacent lanes generated by dideoxy sequencing of pMCT with the MT-CAT oligonucleotide.

Polyclonal pools of stably transfected L cells containing each construct (and controls containing the plasmid $\mathrm{pBS}^{-}$) were made. Primer extension analysis was performed with a transgene-specific CAT primer on total RNA from three independent transformant pools for each construct (Fig. 5 and data not shown). As expected, the wild-type MT-Ipromoted transgene produced hybrid MT-CAT RNAs which paralleled the endogenous MT-I RNAs in 5'-end heterogeneity and +1 RNA predominance (Fig. 5, lanes 5 and 6). The +1 RNA species was induced by metal, and the quantitatively minor upstream RNAs were slightly repressed. In sharp contrast, the TATA mutant transgene produced predominantly the upstream RNAs (Fig. 5, lanes 3 and 4). Some RNA still arose from the +1 region, but it exhibited 5 '-end heterogeneity, a common result of eliminating the TATA element from a TATA-dependent promoter. These data confirm that, in $\mathrm{L}$ cells, the +1 RNA of the wild-type promoter is TATA dependent and show that the upstream RNAs are TATA independent. In addition to this clear qualitative change in RNA expression pattern, mutation of the TATA element led to an apparent increase in the absolute amount of upstream RNA produced in each transfected pool. This may imply that the TATA-dependent and TATA-independent transcriptional modes compete with each other and that ablation of the TATA element altered that competition. However, such quantitative differences between stably transfected polyclonal cell pools are sometimes seen even when separate pools that contain the same construct are compared. At present we favor the former explanation but cannot rule out the latter.

The central result of these experiments is that, in $\mathrm{L}$ cells, the upstream RNAs are independent of the MT-I TATA element, while the +1 start is TATA dependent. Until such mutations are also analyzed in transgenic mice, we cannot be certain that this is true in other cell types, although it seems likely that it will be. Subtle results of the TATA mutation 
were also observed. The first concerns the upstream RNAs of the TATA mutant, which, like those of the parental gene, were slightly repressed by metal. In contrast, the residual +1 region RNAs of the TATA mutant retained some metal inducibility (Fig. 5, lanes 3 and 4, and data not shown), but the effect was small compared with the degree of metal induction seen in the wild-type construct. Another question was whether the TATA mutation alone would switch the L cell pattern to a precise copy of the germ cell pattern. It did not do so. Given the differences already observed in the details of upstream RNA populations in different tissues, it seemed unlikely that TATA dependence alone dictates the pattern of upstream start site utilization. Close inspection of the TATA mutant RNAs in L cells supported this view; start sites adjacent to +1 (e.g., -5 and -12) were much more pronounced than in germ tissue, and the shorter upstream RNAs (e.g., -28 and -44) were much more prevalent than the longer upstream RNAs, the usual L cell pattern. Since the TATA mutation does not result in a complete recapitulation of the germ cell RNA pattern in L cells, the unique germ cell pattern must be created by more than an inability of the TATA element to function. Nonetheless, it is likely that the upstream RNAs in germ cells will share the independence of the TATA element of their $L$ cell counterparts.

Mutation of the distal Sp1 site did not have a major impact on RNA production (Fig. 5, lanes 7 and 8). Therefore, in L cells, this element is not essential for basal expression of either the +1 or the upstream MT-I RNAs and is not required for metal induction of the MT-I +1 RNA. In the transfected pools, expression from the endogenous MT-I gene was not affected by the presence of the various transgenes (data not shown), eliminating the possibility that the behavior of the introduced genes could be due to a promoter titration effect.

\section{DISCUSSION}

We have shown that the mouse MT-I promoter directs RNA transcription via both TATA-dependent and TATAindependent mechanisms, that it functions in both ways even in apparently homogeneous populations of cells, and that the choice between modes is developmentally regulated. This type of compound promoter behavior has not, to our knowledge, been recognized previously. Whether the two modes coexist temporally within a single cell or even at a single promoter is an interesting but unanswered question. A different type of compound promoter has been described for $S$. cerevisiae $(2,24,51)$. Those promoters are compound in the sense that they possess multiple, functionally distinct TATA elements and therefore operate via multiple TATAdependent mechanisms.

We were led to this discovery by the observation that the MT-I promoter initiates transcription at many sites and thus produces multiple species of MT-I transcript. The wellcharacterized +1 transcript (group A in Fig. 2) is initiated 23 nucleotides downstream of the TATA element and is TATA dependent. The newly identified MT-I RNA species (groups $B$ through $F$ in Fig. 2) are initiated at a multitude of sites upstream of the +1 site (most of these are initiated upstream of the TATA element as well) and are, in contrast, TATA independent. Both classes of RNA are properly spliced and polyadenylated. These characteristics strongly argue that both upstream and +1 species are RNA polymerase II products, although this has not yet been determined directly.

The RNAs produced from the two classes of transcription have distinct patterns of tissue-specific expression, implying significant developmental regulation of the choice between TATA-independent and TATA-dependent transcription. While the +1 transcript constitutes the bulk of MT-I RNA in most cell types, in the late meiotic and postmeiotic cells of the testis the TATA-independent transcripts are the predominant MT-I RNA species. In addition, cell types differ in the specific sets of TATA-independent RNAs that they contain, suggesting an additional level of tissue specificity beyond the choice of TATA-independent versus TATA-dependent initiation. For example, approximately $60 \%$ of the TATA-independent RNAs in testis are group D, E, and F RNAs (Fig. 2), but these RNAs contribute only a minor fraction of TATAindependent RNAs in kidney and $L$ cells. The precise locations of the start sites differ as well and fall into two patterns, one epitomized by testis (Fig. 1C, lanes 1 and 2, and data not shown) and the other by kidney (Fig. 1C, lanes 3 and 4 , and data not shown).

The TATA-dependent and TATA-independent classes of RNA are also regulated differently by metal, which is known to increase the overall rate of transcription of the MT-I gene in many cell types (13). Thus, the choice of core promoter mechanism appears to be intimately linked with the gene's metal inducibility. The +1 RNA is metal inducible in all cellular contexts examined except for the testis, which does not exhibit significant metal induction of any MT-I or MT-II RNAs. The TATA-independent RNAs are not induced by metal in any cellular context tested, nor do they become metal responsive in the case of a TATA-minus mutant assayed in cells in which the wild type MT-I gene is metal responsive. We favor the view that these differences in expression of MT-I RNAs are generated mainly at the transcriptional level. However, in the absence of direct measurement of transcription initiation, we cannot exclude the possibility that any of these observations are substantially due to posttranscriptional effects. For example, it is formally possible that preferential stabilization of the upstream RNAs in testis combined with efficient degradation of +1 RNAs in the same cells could give rise to the observed distribution of transcripts. Conversely, preferential destabilization of upstream RNAs in response to metal could be invoked to explain the metal-refractory nature of the upstream RNAs. In the latter case, our studies of MT-I fusion genes show that such differential posttranscriptional effects would have to rely on nucleotides in the -1 to -12 region. Furthermore, these sequences would have to perform any posttranscriptional, metal-responsive function independently of the MT-I sequences replaced by CAT in the transgenes. Such a scenario seems unduly perverse.

The extent of interrelation between TATA-dependent and TATA-independent transcriptional modes is an issue raised but not resolved by our observations. Closely related DNAprotein complexes could activate both transcriptional modes, with a subset of components determining the balance and separate characteristics of TATA dependence and TATA independence. Alternatively, mutually exclusive complexes containing entirely different accessory factors could be used. The ability of promoters which normally appear to support little or no TATA-independent transcription to function after TATA element removal $(3,5,12,17$, 19) and the ability of Sp1 to stimulate both TATA-dependent and TATA-independent transcription (14) argue that the switch to TATA-independent transcription may not require wholesale remodeling of the transcription complex.

It is interesting to consider this new information about MT-I promoter function in the context of what is known of its structure. The MT-I proximal regulatory region contains 
a number of cis-acting elements identified either genetically or by homology to known transcription factor-binding sites (Fig. 2B). These include several metal-responsive enhancer elements (MREs) $(7,49,52)$ and potential binding sites for general transcription factors such as $\operatorname{MLTF}(8,10,46)$ and Sp1 (28). In vivo footprinting in L cells has detected DNAprotein interactions at many of these sites, with only the MRE interactions dependent on metal treatment (39) (Fig. 2B legend). The relevance of this current picture of MT-I DNA-transcription factor interactions to the TATA-independent mode of transcription is unknown, but it is of interest that the MREs directly overlap a number of the upstream start sites. Metal-dependent occupancy of these sites activates transcription only from the +1 region, suggesting that they are either inappropriately positioned to activate upstream RNA synthesis or incompatible with the upstream initiation complexes. The MREs could also be responsible for the observed metal-induced decrease in upstream RNA in $\mathrm{L}$ cells. Factors bound to the MREs could suppress the use of the upstream start sites directly, by steric occlusion, or indirectly, by activation of the TATA-dependent transcriptional mode at the expense of the TATA-independent mode.

Further investigation of these phenomena is likely to be difficult using $\mathrm{L}$ cells alone, considering the small amount of upstream RNA that they contain. However, an in vivo footprint study of the MT-I gene in the pachytene cells of the testis together with analysis of site-directed mutants in the testes and other tissues of transgenic mice may provide further insight into regulation of this compound promoter. Ultimately, the MT-I compound promoter should serve as a model system for in vitro reconstruction of promoter choice and for characterization of the specific protein interactions that account for the strongly preferential interaction of the metal response with TATA-dependent initiation.

\section{ACKNOWLEDGMENTS}

We thank Kelly Thomas, Mel Simon, and Debra Wolgemuth for generous gifts of purified testis cell-type RNAs and mutant RNAs, Richard Palmiter for pMT-I $\Delta \mathrm{i}$, Jim Maher for assistance with mutagenesis, Sean Tavtigian for his initial observations, Tom Novak for the BB62, Paul Mueller, Jeff Miner, and members of the Wold lab for materials and invaluable discussion, and David Anderson, Richard Axel, and Mel Simon for reading the manuscript.

This work was supported by grants from the National Institutes of Health, Rita Allen Foundation, and Lucille P. Markey Charitable Trust to B.W. and a National Research Service Award (5 T32 GM 07616-11) from the National Institute of General Medical Sciences to P.G.

\section{LITERATURE CITED}

1. Angel, P., M. Imagawa, R. Chiu, B. Stein, R. J. Imbra, H. J. Rahmsdorf, C. Jonat, P. Herrlich, and M. Karin. 1987. Phorbol ester-inducible genes contain a common cis element recognized by a TPA-modulated trans-acting factor. Cell 49:729-739.

2. Arndt, K. T., C. Styles, and G. R. Fink. 1987. Multiple global regulators control HIS4 transcription in yeast. Science 237:874 880.

3. Benoist, C., and P. Chambon. 1981. In vivo sequence requirements of the SV40 early promoter region. Nature (London) 290:304-315.

4. Brandhorst, B. P., and E. H. McConkey. 1974. Stability of nuclear RNA in mammalian cells. J. Mol. Biol. 85:451-463.

5. Breathnach, R., and P. Chambon. 1981. Organization and expression of eukaryotic split genes coding for proteins. Annu. Rev. Biochem. 50:349-383.

6. Buratowski, S., S. Hahn, P. A. Sharp, and L. Guarente. 1988. Function of a yeast TATA element-binding protein in a mam- malian transcription system. Nature (London) 334:37-42.

7. Carter, A. D., B. K. Felber, M. Walling, M.-F. Jubier, C. J. Schmidt, and D. H. Hamer. 1984. Duplicated heavy metal control sequences of the mouse metallothionein-I gene. Proc. Natl. Acad. Sci. USA 81:7392-7396.

8. Carthew, R. W., L. A. Chodosh, and P. A. Sharp. 1985. An RNA polymerase II transcription factor binds to an upstream element in the adenovirus major late promoter. Cell 43:439-448.

9. Cathala, G., J.-F. Savouret, B. Mendez, B. L. West, M. Karin, J. A. Martial, and J. D. Baxter. 1983. Laboratory methods-a method for isolation of intact, translationally active ribonucleic acid. DNA 2:329-335.

10. Chodosh, L. A., R. W. Carthew, and P. A. Sharp. 1986. A single polypeptide possesses the binding and transcription activities of the adenovirus major late transcription factor. Mol. Cell. Biol. 6:4723-4733.

11. Chomczynski, P., and N. Sacchi. 1987. Single-step method of RNA isolation by acid guanidinium thiocyanate-phenol-chloroform extraction. Anal. Biochem. 162:156-159.

12. Dierks, P., A. van Ooyen, M. D. Cochran, C. Dobkin, J. Reiser, and C. Weissman. 1983. Three regions upstream from the cap site are required for efficient and accurate transcription of the rabbit $\beta$-globin gene in mouse 3T6 cells. Cell 32:695-706.

13. Durnam, D. M., and R. D. Palmiter. 1981. Transcriptional regulation of the mouse metallothionein-I gene by heavy metals. J. Biol. Chem. 256:5712-5716.

14. Dynan, W. S., S. Sazer, R. Tjian, and R. T. Schimke. 1986. Transcription factor $\mathrm{Spl}$ recognises a DNA sequence in the mouse dihydrofolate reductase promoter. Nature (London) 319: 246-248.

15. Eisenmann, D. M., C. Dollard, and F. Winston. 1989. SPT15, the gene encoding the yeast TATA binding factor TFIID, is required for normal transcription in yeast. Cell 58:1183-1191.

16. Farnham, P. J., and R. T. Schimke. 1986. Murine dihydrofolate reductase transcripts through the cell cycle. Mol. Cell. Biol. 6:365-371.

17. Ghosh, P. K., P. Lebowitz, R. J. Frisque, and Y. Gluzman. 1981. Identification of a promoter component involved in positioning the 5 ' termini of simian virus 40 early mRNAs. Proc. Natl. Acad. Sci. USA 78:100-104.

18. Glanville, N., D. M. Durnam, and R. D. Palmiter. 1981. Structure of mouse metallothionein-I gene and its mRNA. Nature (London) 292:267-269.

19. Grosschedl, R., and M. L. Birnstiel. 1980. Identification of regulatory sequences in the prelude sequences of an HSA histone gene by the study of specific deletion mutants in vivo. Proc. Natl. Acad. Sci. USA 77:1432-1436.

20. Gunn, S. A., T. C. Gould, and W. A. D. Anderson. 1965. Strain differences in susceptibility of mice and rats to cadmiuminduced testicular damage. J. Reprod. Fertil. 10:273-275.

21. Hahn, S., S. Buratowski, P. A. Sharp, and L. Guarente. 1989. Yeast TATA-binding protein TFIID binds to TATA elements with both consensus and nonconsensus DNA sequences. Proc. Natl. Acad. Sci. USA 86:5718-5722.

22. Hahn, S., S. Buratowski, P. A. Sharp, and L. Guarente. 1989. Isolation of the gene encoding the yeast TATA-binding protein TFIID: a gene identical to the SPT15 suppressor of Ty element insertions. Cell 58:1173-1181.

23. Hamer, D. H. 1986. Metallothionein. Annu. Rev. Biochem. 55:913-951.

24. Harbury, P. A. B., and K. Struhl. 1989. Functional distinctions between yeast TATA elements. Mol. Cell. Biol. 9:5298-5304.

25. Horikoshi, M., C. K. Wang, H. Fujii, J. A. Cromlish, P. A. Weil, and R. G. Roeder. 1989. Cloning and structure of a yeast gene encoding a general transcription initiation factor TFIID that binds to the TATA box. Nature (London) 341:299-303.

26. Ishii, S., Y. Xu, R. H. Stratton, B. A. Roe, G. T. Merlino, and I. Pastan. 1985. Characterization and sequence of the promoter region of the human epidermal growth factor receptor gene. Proc. Natl. Acad. Sci. USA 82:4920-4924.

27. Jones, K. A., K. R. Yamamoto, and R. Tjian. 1985. Two distinct transcription factors bind to the HSV thymidine kinase promoter in vitro. Cell 42:559-572. 
28. Kadonaga, J. T., K. A. Jones, and R. Tjian. 1986. Promoterspecific activation of RNA polymerase II transcription by Spl. Trends Biochem. Sci. 11:20-23.

29. Kim, S. K., and B. J. Wold. 1985. Stable reduction of thymidine kinase activity in cells expressing high levels of anti-sense RNA. Cell 42:129-138.

30. Maher, L. J., B. Wold, and P. B. Dervan. 1989. Inhibition of DNA-binding proteins by oligonucleotide-directed triple helix formation. Science 245:725-730.

31. McGrogan, M., C. C. Simonsen, D. T. Smouse, P. J. Farnham, and R. T. Schimke. 1985. Heterogeneity at the $5^{\prime}$ termini of mouse dihydrofolate reductase mRNAs. J. Biol. Chem. 260: 2307-2314.

32. McKnight, S. L., E. R. Gavis, R. Kingsbury, and R. Axel. 1981. Analysis of transcriptional regulatory signals of the HSV thymidine kinase gene: identification of an upstream control region. Cell 25:385-398.

33. Means, A. L., and P. J. Farnham. 1990. Transcription initiation from the dihydrofolate reductase promoter is positioned by housekeeping initiation protein 1 binding at the initiation site. Mol. Cell. Biol. 10:653-661.

34. Meek, E. S. 1959. Cellular changes induced by cadmium in mouse testis and liver. Br. J. Exp. Pathol. 40:503-507.

35. Melton, D. W., C. McEwan, A. B. McKie, and A. M. Reid. 1986. Expression of the mouse HPRT gene: deletional analysis of the promoter region of an X-chromosome linked housekeeping gene. Cell 44:319-328.

36. Mintz, B., and E. S. Russell. 1957. Gene-induced embryological modifications of primordial germ cells in the mouse. J. Exp. Zool. 134:207-230.

37. Mitchell, P. J., A. M. Carothers, J. H. Han, J. D. Harding, E. Kas, L. Venola, and L. A. Chasin. 1986. Multiple transcription start sites, DNase I-hypersensitive sites, and an opposite-strand exon in the $5^{\prime}$ region of the $\mathrm{CHO} d h f r$ gene. Mol. Cell. Biol. 6:425-440.

38. Mitchell, P. J., and R. Tjian. 1989. Transcriptional regulation in mammalian cells by sequence-specific DNA-binding proteins. Science 245:371-378.

39. Mueller, P. R., S. J. Salser, and B. Wold. 1988. Constitutive and metal-inducible protein-DNA interactions at the mouse metallothionein I promoter examined by in vivo and in vitro footprinting. Genes Dev. 2:412-427.

40. Nakajima, N., M. Horikoshi, and R. G. Roeder. 1988. Factors involved in specific transcription by mammalian RNA polymerase II: purification, genetic specificity, and TATA box-promoter interactions of TFIID. Mol. Cell. Biol. 8:4028-4040.

41. Nocka, K., S. Majumdar, B. Chabot, P. Ray, M. Cervone, A. Bernstein, and P. Besmer. 1989. Expression of c-kit gene products in known cellular targets of $\mathrm{W}$ mutations in normal and $\mathrm{W}$ mutant mice: evidence for an impaired c-kit kinase in mutant mice. Genes Dev. 3:816-826.

42. Nordberg, G. F. 1971. Effects of acute and chronic cadmium exposure on the testicles of mice. Environ. Physiol. 1:171-187.

43. Parizek, J. 1960. Sterilization of the male by cadmium salts. J. Reprod. Fertil. 1:294-309.

44. Reynolds, G. A., S. K. Basu, T. F. Osborne, D. J. Chin, G. Gil, M. S. Brown, J. L. Goldstein, and K. L. Luskey. 1984. HMG CoA reductase: a negatively regulated gene with unusual promoter and 5' untranslated regions. Cell 38:275-285.

45. Sassone-Corsi, P., J. Corden, and P. Chambon. 1981. Promotion of specific in vitro transcription by excised "TATA" box sequences inserted in a foreign nucleotide environment. Nucleic Acids Res. 9:3941-3958.

46. Sawadogo, M., and R. G. Roeder. 1985. Interaction of a genespecific transcription factor with the adenovirus major late promoter upstream of the TATA box region. Cell 43:165-175.

47. Sazer, S., and R. T. Schimke. 1986. A re-examination of the 5 termini of mouse dihydrofolate reductase RNA. J. Biol. Chem. 261:4685-4690.

48. Searle, P. F., B. L. Davison, G. W. Stuart, T. M. Wilkie, G. Norstedt, and R. D. Palmiter. 1984. Regulation, linkage, and sequence of mouse metallothionein I and II genes. Mol. Cell. Biol. 4:1221-1230.

49. Searle, P. F., G. W. Stuart, and R. D. Palmiter. 1985. Building a metal-responsive promoter with synthetic regulatory elements. Mol. Cell. Biol. 5:1480-1489.

50. Smale, S. T., and D. Baltimore. 1989. The "initiator" as a transcription control element. Cell 57:103-113.

51. Struhl, K. 1986. Constitutive and inducible Saccharomyces cerevisiae promoters: evidence for two distinct molecular mechanisms. Mol. Cell. Biol. 6:3847-3853.

52. Stuart, G. W., P. F. Searle, H. Y. Chen, R. L. Brinster, and R. D. Palmiter. 1984. A 12-base-pair DNA motif that is repeated several times in metallothionein gene promoters confers metal regulation to a heterologous gene. Proc. Natl. Acad. Sci. USA 81:7318-7322

53. Thomas, K. H., T. M. Wilkie, P. Tomashefsky, A. R. Bellve, and M. I. Simon. 1989. Differential gene expression during mouse spermatogenesis. Biol. Reprod. 41:729-739.

54. Wolgemuth, D. J., E. Gizang-Ginsberg, E. Engelmyer, B. J. Gavin, and C. Ponzetto. 1985. Separation of mouse testis cells on a Celsep ${ }^{\mathrm{TM}}$ apparatus and their usefulness as a source of high-molecular-weight DNA or RNA. Gamete Res. 12:1-10.

55. Zakeri, Z. F., and D. J. Wolgemuth. 1987. Development-stagespecific expression of the hsp70 gene family during differentiation of the mammalian male germ line. Mol. Cell. Biol. 7:17911796.

56. Zinn, K. A., D. DiMaio, and T. Maniatis. 1983. Identification of two distinct regulatory regions adjacent to the human $\beta$-interferon gene. Cell 34:865-879. 